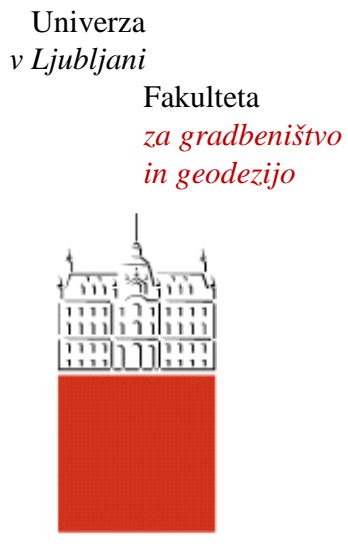

Jamova 2

1000 Ljubljana, Slovenija http://www3.fgg.uni-lj.si/

DRUGG - Digitalni repozitorij UL FGG http://drugg.fgg.uni-lj.si/

Ta članek je avtorjeva zadnja recenzirana različica, kot je bila sprejeta po opravljeni recenziji.

Prosimo, da se pri navajanju sklicujete na bibliografske podatke, kot je navedeno:

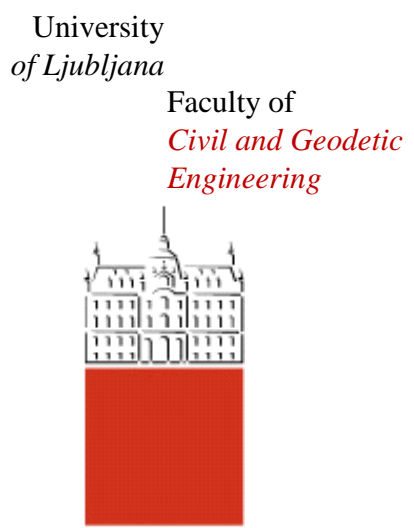

Jamova 2

SI - 1000 Ljubljana, Slovenia http://www3.fgg.uni-lj.si/en/

DRUGG - The Digital Repository http://drugg.fgg.uni-lj.si/

This version of the article is author's manuscript as accepted for publishing after the review process.

When citing, please refer to the publisher's bibliographic information as follows:

Marjetič, A., Ambrožič, T., Turk, G., Sterle, O. in Stopar, B. 2010. Statistical Properties of Strain and Rotation Tensors in Geodetic Network. Journal of Surveying Engineering ASCE, 136, 3: 102-110.

DOI: 10.1061/(ASCE)SU.1943-5428.0000020. 


\section{Editorial Manager(tm) for Journal of Surveying Engineering}

Manuscript Draft

Manuscript Number: SUENG-48R2

Title: STATISTICAL PROPERTIES OF STRAIN AND ROTATION TENSORS IN GEODETIC NETWORK

Article Type: Technical Paper

Corresponding Author: Mr. Aleš Marjetič,

Corresponding Author's Institution: University of Ljubljana

First Author: Aleš Marjetič

Order of Authors: Aleš Marjetič; Tomaž Ambrožič, Ph.D.; Goran Turk, Ph.D.; Oskar Sterle, M.S.; Bojan Stopar, Ph.D.

Abstract: This article deals with the characteristics of deformation of a body or a figure represented by discrete points of geodetic network. In each point of geodetic network kinematic quantities are considered: normal strain, shear strain and rotation. They are computed from strain and rotation tensors represented by displacement gradient matrix on the basis of known point displacement vector. Deformation analysis requires the appropriate treatment of kinematic quantities. Thus statistical properties of each quantity in a single point of geodetic network have to be known. Empirical results have shown that statistical properties are strongly related to the orientation in single point and local geometry of the geodetic network. Based on the known probability distribution of kinematic quantities the confidence areas for each quantity in certain point can be defined. Based on this we can carry out appropriate statistical testing and decide whether the deformation of network in each point is statistically significant or not. On the other hand, we are able to ascertain the quality of the geometry of the geodetic network. Known characteristics of the probability distributions of two strain parameters and rotation in each point can serve us as useful tool in the procedures of optimizing the geometry of the geodetic networks.

Suggested Reviewers:

Opposed Reviewers: 
Dear Editor!

We would like to thank the reviewers for their comments and suggestions. In Response to reviewers comments we summarize all comments, suggestions and errors concerning our manuscript. For each comment we marked it as correction done or gave detailed explanation why we disagree with the reviewer comment.

Sincerely,

Authors 
Click here to download Manuscript: manuscript_rev_bw1.doc

\title{
STATISTICAL PROPERTIES OF STRAIN AND ROTATION TENSORS IN GEODETIC NETWORK
}

Aleš Marjetičํㅜ Tomaž Ambrožič², Goran Turk ${ }^{3}$, Oskar Sterle ${ }^{4}$ and Bojan Stopar ${ }^{5}$

\begin{abstract}
This article deals with the characteristics of deformation of a body or a figure represented by discrete points of geodetic network. In each point of geodetic network kinematic quantities are considered: normal strain, shear strain and rotation. They are computed from strain and rotation tensors represented by displacement gradient matrix on the basis of known point displacement vector. Deformation analysis requires the appropriate treatment of kinematic quantities. Thus statistical properties of each quantity in a single point of geodetic network have to be known. Empirical results have shown that statistical properties are strongly related to the orientation in single point and local geometry of the geodetic network. Based on the known probability distribution of kinematic quantities the confidence areas for each quantity in certain point can be defined. Based on this we can carry out appropriate statistical testing and decide whether the deformation of network in each point is statistically significant or not. On the other hand, we are able to ascertain the quality of the geometry of the geodetic network. Known characteristics of the probability distributions of two strain parameters and rotation in each point can serve us as useful tool in the procedures of optimizing the geometry of the geodetic networks.
\end{abstract}

KEY WORDS: kinematic quantities; strain; rotation; tensor; normal strain; shear strain; geodetic network; probability distribution; confidence interval; confidence area; detection level of deformations

\footnotetext{
${ }^{1}$ assistant, Faculty of Civil and Geodetic Engineering, Univ. of Ljubljana, Jamova cesta 2, 1000 Ljubljana, Slovenia. E-mail: amarjeti@fgg.uni-lj.si

2 associate professor, Faculty of Civil and Geodetic Engineering, Univ. of Ljubljana, Jamova cesta 2, 1000

Ljubljana, Slovenia. E-mail: tambrozi@fgg.uni-lj.si

3 professor, Faculty of Civil and Geodetic Engineering, Univ. of Ljubljana, Jamova cesta 2, 1000 Ljubljana,

Slovenia. E-mail: gturk@fgg.uni-lj.si

${ }^{4}$ assistant, Faculty of Civil and Geodetic Engineering, Univ. of Ljubljana, Jamova cesta 2, 1000 Ljubljana, Slovenia.

E-mail: osterle@fgg.uni-lj.si

5 associate professor, Faculty of Civil and Geodetic Engineering, Univ. of Ljubljana, Jamova cesta 2, 1000

Ljubljana, Slovenia. E-mail: bstopar@fgg.uni-lj.si
} 


\section{INTRODUCTION}

Deformations are generally determined on the basis of the displacements of individual points of a deformable body represented by geodetic network. In this paper we will use a slightly different approach in determining statistically significant deformations in the geodetic network according to classical deformation analysis methods (Delft, Hannover, Karlsruhe, Fredericton... (Chrzanowski et al. 1983; Mierlo 1978; Welsch and Zhang 1983)). Deformation of the body, which is represented by a certain number of points in the geodetic network, will stem from the theory of the mechanics of solids. The values of kinematic quantities, normal strain, shear strain and rotation, which are calculated from displacement gradient matrix, will be treated. According to the known classical methods of deformation analysis, our focus is on finding a statistically significant deformation in each point. In order to perform the appropriate statistical testing for determing the statistical significance of deformations, we need information about the probability distribution of a particular kinematic quantity. The aim is therefore to provide the probability distribution of each strain parameter and rotation in each point and direction of geodetic network. Some researchers treated them as normally distributed random variables (Michel and Person 2003). The probability distributions of kinematic quantities as eigenspace components (principal eigenvalues) of the rank-two random tensor are significantly different from commonly used Gauss normal distribution (Cai et al. 2005; Cai and Grafarend 2007a/b; Xu and Grafarend 1996). The problem was already intensively studied and the probability properties of eigenvalues of rank-two random tensor which components are supposed to be normally distributed was analytically derived (Soler and Gelder 1991; Xu and Grafarend 1996; Cai et al. 2005). Even though the analytical solutions exists for linearized theory, we decided to use the empirical approach. As mechanical strains and rotations are in the functional relationship with the point displacements and therefore with the geodetic observations too, we are able to empirically determine the probability distribution of each quantity by varying the value of observations in geodetic network within their own confidence intervals. As is known in statistics, by identifying the characteristics of a sample of random variable, we can calculate the mean values and boundaries of confidence intervals at selected confidence level. These values allow us to determine statistical significance of deformation at a single point in the geodetic network. The size of the confidence interval of each kinematic quantity is unique for each point in the geodetic network and as will be shown also depends on the directon in which it is treated. So the term confidence interval will be replaced with the term confidence area. Comparison of the shapes and sizes of confidence areas at different points allows us to determine the parts of the network, where smaller deformations are detectable. On the other hand, we 


\section{Assumption of homogenous strains and rotation}

Definition of strain and rotation tensor is based on the displacement vector $\mathbf{u}(x, y)=\left[\begin{array}{ll}u_{x} & u_{y}\end{array}\right]^{\mathrm{T}}$ for each point of the considered deformable body, where generally heterogeneous strains and rotations occur. In the case of geodetic network, we have a situation where informations about displacements only in some specific points of the inspected body are available. In this case we can determine the displacements and consequently the strain and rotation tensor only in geodetic points. In some arbitrary point, strain and rotation tensor is determined through various methods of 
interpolation under the assumption of homogeneous deformations in certain areas where strains and rotations are constants. Such areas are e.g. triangles obtained by Deulanay triangulation (Cai and Grafarend 2007a, 2007b).

In all cases we consider tensor of small strains and small rotations because the deformation of the object is assumed differentially small compared to the dimension of the object, represented by the geodetic network. Using that and the fact that the displacement vector and the associated vector field are in the case of homogeneous strains and rotations continuous, differentiable and regular, the displacement vector is totally differentiable and is connected with the displacement gradient matrix $\mathbf{E}_{i}$ in relation (Shames and Cozzarelli 1997; Vaniček et al. 2001; Vaniček et al. 2008; Berber 2006; Berber et al. 2006):

$$
\mathbf{u}\left(T_{j}\right)=\mathbf{E}_{i} \cdot \mathbf{r}_{i j}+\mathbf{c},
$$

$$
\begin{aligned}
& \text { where: } \mathbf{E}_{i}=\left[\begin{array}{ll}
\frac{\partial u_{x}}{\partial x} & \frac{\partial u_{x}}{\partial y} \\
\frac{\partial u_{y}}{\partial x} & \frac{\partial u_{y}}{\partial y}
\end{array}\right] \text { - displacement gradient matrix for point } T_{i}, \\
& \mathbf{r}_{i j}=\mathbf{r}_{j}-\mathbf{r}_{i}=\left[\begin{array}{c}
x_{T_{j}}-x_{T_{i}} \\
y_{T_{j}}-y_{T_{i}}
\end{array}\right] \text { - coordinate differences of points } T_{i} \text { and } T_{j} \\
& \mathbf{c}=\left[\begin{array}{l}
u_{x}\left(T_{i}\right) \\
u_{y}\left(T_{i}\right)
\end{array}\right]=\left[\begin{array}{l}
a_{i} \\
b_{i}
\end{array}\right]-\text { constant vector. }
\end{aligned}
$$

If the system of equations (1) is written in matrix form, we get (where indices for points are simplified with $i$ and j):

$$
\begin{aligned}
& \mathrm{F}_{j, 1}:\left[\begin{array}{lll}
1 & \left(x_{j}-x_{i}\right) & \left(y_{j}-y_{i}\right)
\end{array}\right]\left[\begin{array}{lll}
a_{i} & \frac{\partial u_{x i}}{\partial x} & \frac{\partial u_{x i}}{\partial y}
\end{array}\right]^{\mathrm{T}}=u_{x}\left(T_{j}\right), \\
& \mathrm{F}_{j, 2}:\left[\begin{array}{lll}
1 & \left(x_{j}-x_{i}\right) & \left(y_{j}-y_{i}\right)
\end{array}\right]\left[\begin{array}{lll}
b_{i} & \frac{\partial u_{y i}}{\partial x} & \frac{\partial u_{y i}}{\partial y}
\end{array}\right]^{\mathrm{T}}=u_{y}\left(T_{j}\right) .
\end{aligned}
$$

$$
\begin{aligned}
& \text { Primarily equations (1) and (2) represent a change of vector field of displacement along the connection between } T_{i} \\
& \text { in } T_{j} \text { according to the starting point } T_{i} \text {. In the case of the assumptions of homogeneous strains and rotation, } \\
& \text { displacement vector field varies linearly between two points. Here it is important to emphasize that such equations }
\end{aligned}
$$


are only valid for the connections between points in the geodetic network, which are located on the area of supposed homogenous strains and rotations. This is logical, since it is difficult to speak about the correlation between the strains of distant points.

System of equations (2) represents the system of two linear equations for unknowns $a_{i}, \frac{\partial u_{x i}}{\partial x}, \frac{\partial u_{x i}}{\partial y}, b_{i}, \frac{\partial u_{y i}}{\partial x}, \frac{\partial u_{y i}}{\partial y}$. If the system (2) is written for all neighbouring points $j=0,1, \ldots, k$ to point $i$ in matrix form, we get (Cai and Grafarend 2007a, 2007b):

$$
\left[\begin{array}{cccccc}
1 & \left(x_{1}-x_{i}\right) & \left(y_{1}-y_{i}\right) & 0 & 0 & 0 \\
0 & 0 & 0 & 1 & \left(x_{1}-x_{i}\right) & \left(y_{1}-y_{i}\right) \\
1 & \left(x_{2}-x_{i}\right) & \left(y_{2}-y_{i}\right) & 0 & 0 & 0 \\
0 & 0 & 0 & 1 & \left(x_{2}-x_{i}\right) & \left(y_{2}-y_{i}\right) \\
\vdots & \vdots & \vdots & \vdots & \vdots & \vdots \\
1 & \left(x_{k}-x_{i}\right) & \left(y_{k}-y_{i}\right) & 0 & 0 & 0 \\
0 & 0 & 0 & 1 & \left(x_{k}-x_{i}\right) & \left(y_{k}-y_{i}\right) \\
1 & 0 & 0 & 0 & 0 & 0 \\
0 & 0 & 0 & 1 & 0 & 0
\end{array}\right] \cdot\left[\begin{array}{c}
a_{i} \\
\frac{\partial u_{x i}}{\partial x} \\
\frac{\partial u_{x i}}{\partial y} \\
b_{i} \\
\frac{\partial u_{y i}}{\partial x} \\
\partial u_{y i} \\
\partial y
\end{array}\right]=\left[\begin{array}{c}
u_{x 1} \\
u_{y 1} \\
u_{x 2} \\
u_{y 2} \\
\vdots \\
u_{x k} \\
u_{y k} \\
u_{x i} \\
u_{y i}
\end{array}\right],
$$

or:

$$
\mathbf{F} \cdot \operatorname{vec}\left(\mathbf{E}_{i}^{*}\right)=\mathbf{u}_{j}
$$

with $\mathbf{F}$ - design matrix, $\mathbf{u}_{j}$ - vector of point displacement obtained by the least squares adjustment of observations and $\operatorname{vec}\left(\mathbf{E}_{i} *\right)$ - displacement gradient matrix written in vector mode, expanded for the constant elements $a_{i}$ and $b_{i}$. The solution for $\operatorname{vec}\left(\mathbf{E}_{i} *\right)$ is computed from overdetermined system (3) and (4) by the least squares method:

$$
\operatorname{vec}\left(\mathbf{E}_{i}^{*}\right)=\left(\mathbf{F}^{\mathrm{T}} \mathbf{P}_{F} \mathbf{F}\right)^{-1} \mathbf{F}^{\mathrm{T}} \mathbf{P}_{F} \cdot \mathbf{u}_{j}
$$

Because distances between point $T_{i}$ and neighbouring points vary, strains and rotations do not have equal impact on the strains and rotations in point $T_{i}$. The longer the distance between two neighbouring points, the smaller the impact on the strains and rotations in point $T_{i}$ can be expected. Because design matrix $\mathbf{F}$ just connects deformation parameters in $T_{i}$ with point displacements of $T_{j}$ and does not provide the influence of different distance between points on deformations in point $T_{i}$ it is reasonable to use the diagonal weight matrix $\mathbf{P}_{F}$. It gives the proper treatment of the deformation influence of distant points. The weights in $\mathbf{P}_{F}$ can be defined as inverse square of 
distance $d_{i j}$ between two points, in order to further reduce the impact of strains and rotations occurred at distant points. The denominator is expanded with constant 1 to avoid infinite values in point $T_{i}\left(d_{i i}=0\right)$ :

$$
p_{i j}=\frac{1}{1+d_{i j}^{2}}
$$

We checked the appropriatness of equation (6) for the weight of each deformation (displacement) in point $T_{j}$ by using the methods of optimization of second order design (Stopar 2001) in the case of selected geodetic network (Figure 2). Primarily, these methods of optimizations are used to determine the optimal measurement weights in the selected geometry of the geodetic network in relation to the desired precision of the unknown coordinates of points, as it is provided by a criterion matrix (Stopar 2001). In our case, we start with the required precision of the elements of displacement gradient matrix $\mathbf{E}_{i}$ in equation (4). By taking into account all the possible connections between all points and single point $T_{i}$ (to construct a matrix $\mathbf{F}$ ) we can optimize weights $p_{i j}$ of each displacement of point $T_{j}$ and looking for functional dependence of the estimated weights on the distance between points. It can be seen from the graph (Figure 1) that the weights are decreasing with the square of the distance between two points. The interpolated trend does not fully coincide with the equation (6) in terms of constants in the numerator. The reason is that automatic interpolation of trend function is because of the configuration of the selected network carried out only for distances larger than $350 \mathrm{~m}$ (shortest distance in network), which may affect the interpolated trend function.

\section{Kinematic quantities in geodetic network}

The displacement gradient matrix $\mathbf{E}_{i}$ can be decomposed into symmetrical part - tensor of small strains ( $\boldsymbol{\varepsilon}$ ) and anti-symmetrical part - tensor of small rotations ( $\boldsymbol{\omega}$ ) (see e.g. Shames and Cozzarelli 1997):

$$
\mathbf{E}_{i}=\left[\begin{array}{cc}
\frac{\partial u_{x}}{\partial x} & \frac{\partial u_{x}}{\partial y} \\
\frac{\partial u_{y}}{\partial x} & \frac{\partial u_{y}}{\partial y}
\end{array}\right]=\left[\begin{array}{cc}
\varepsilon_{x x} & \varepsilon_{x y}+\omega_{z} \\
\varepsilon_{x y}-\omega_{z} & \varepsilon_{y y}
\end{array}\right]=\left[\begin{array}{cc}
\varepsilon_{x x} & \varepsilon_{x y} \\
\varepsilon_{x y} & \varepsilon_{y y}
\end{array}\right]+\left[\begin{array}{cc}
0 & \omega_{i} \\
-\omega_{i} & 0
\end{array}\right]=\boldsymbol{\varepsilon}+\boldsymbol{\omega} .
$$

Since it is reasonable to present the strain and rotaion in point only with the maximum values, we focus only on the principal normal strain and the principle shear strain (see e.g. Shames and Cozzarelli 1997): 
- principal normal strain:

$$
\varepsilon_{1,2}^{i}=\frac{\varepsilon_{x x}+\varepsilon_{y y}}{2} \pm \sqrt{\frac{1}{4}\left(\varepsilon_{x x}-\varepsilon_{y y}\right)^{2}+\varepsilon_{x y}^{2}} \text {, in the direction } \alpha_{\varepsilon_{1}}=\frac{1}{2} \arctan \left(\frac{2 \varepsilon_{x y}}{\varepsilon_{x x}-\varepsilon_{y y}}\right)
$$

- principal shear strain

$$
\gamma_{1,2}^{i}= \pm \sqrt{\frac{1}{4}\left(\varepsilon_{x x}-\varepsilon_{y y}\right)^{2}+\varepsilon_{x y}^{2}} \text {, in the direction } \alpha_{\gamma_{1}}=\frac{1}{2} \arctan \left(-\frac{\varepsilon_{x x}-\varepsilon_{y y}}{2 \varepsilon_{x y}}\right)
$$

- and differential rotation in point $\omega_{i}$.

We discuss only the maximum value of shear strain, because both extreme values differ only in the \pm sign.

The principal normal strain represents the maximum scale deformation, the principal shear strain the deformation of local network configuration and the differential rotation the local rotation of the network in the selected point.

All three kinematic quantities are theoretically datum invariant (Shames and Cozzarelli 1997; Vaniček et al. 2001; Vaniček et al. 2008) and give strain and rotation properties of the deformable body (object or area or figure), which are invariant to the selected coordinate system in which they are treated. Strain and rotation tensors are invariant to the definition of coordinate system so the computed values of strain parameters and rotation in network points are independent of the definition of datum parameters in geodetic network. But it is important to emphasize that the invariance of strains and rotation to datum definition has no relation to the lack of uniformity of geodetic datums between two time epochs. It has been shown (Xu et al. 2000) that unequally defined geodetic datum between two time epochs, despite invariant nature of strains, leads to changes in computed kinematic quantities. With geodetic datum differences we cannot assure the consistency of deformation state in both time epochs and therefore the real point displacement field needed for determination of strains and rotations cannot be computed. In particular, concerning geodetic approach, there is a problem using free network adjustment, when geodetic network is not externally tied to a fixed reference frame (Xu et al. 2000).

In the sequel the article will focus on the study of statistical properties of deformation parameters.

\section{STATISTICAL PROPERTIES OF STRAINS AND ROTATIONS}


The statistical properties of strain and rotation tensor are represented by the probability distribution of appertaining kinematic quantities considered as random variables. Kinematic quantities, derived from strain and rotation tensor, are in a nonlinear functional relation with the point displacements in the network (equations (1) and (7)) occurring between different time epochs. This is why the analytical derivation of probability distribution functions is very complex (Soler and Van Gelder 1991, 2006; Xu and Grafarend 1996; Cai et al. 2005). Empirical determination of the probability distribution of each kinematic quantity could be therefore more appropriate.

The probability distribution of kinematic quantities in geodetic network can be empirically derived by simulated observations in the network using Monte Carlo approach (Rubinstein 1981). Simulated observations are adjusted using the least square method to obtain the vector of coordinate unknowns or corrections to approximate coordinates of points. In this case we consider corrections of coordinates as random displacements of points, which are used to determine the strain and rotation tensor for each of these artificially generated displacements. The strain and rotation tensor can be then characterized by three values of kinematic quantities in each point. In this case we can determine how the network responds to any random change in observations within its confidence interval in relation to precision of used geodetic instruments and measurements methods.

Statistical properties of kinematic quantities as random variable can be expressed by its probability distribution function or the first and second order moments of random variable (mean value and standard deviation). The number of simulated sets of observations (sim) is the sample size of computed kinematic quantities with their own directions (equations (8) and (9) - eigendirections of strain tensor) in particular point. Considering the fact that the deformable body, represented here by geodetic network, responds differently to the strains influencing in different directions, it is important to study the statistical behaviour of each kinematic quantity in certain point as function of orientation. Overall consideration, as e.g. made by Michel and Person (2003), without taking into account orientation of deformations cannot be complete except for differential rotation in point.

From above it is necessary to divide the whole sample of computed kinematic quantities (for particular point) into groups belonging to different directions or from numerical aspect the direction intervals - sectors. For each sector with the sample size $\operatorname{sim}_{s e c}$ of computed kinematic quantities the mean value of each quantity and its standard deviation for each point in the geodetic network can be computed as: 
Mean values and their standard deviations determine the limits of confidence interval at the chosen level of confidence $1-\alpha$. For the chosen $\alpha$ the confidence interval includes $1-\alpha$ of all values in sample of certain size.

$$
\varepsilon_{\text {sec }}^{\mathrm{det}}=\bar{\varepsilon}_{\text {sec }} \pm C V_{\text {sec }}^{\varepsilon} \cdot s_{\text {sec }}^{\varepsilon}, \gamma_{s e c}^{\mathrm{det}}=\bar{\gamma}_{\text {sec }} \pm C V_{\text {sec }}^{\gamma} \cdot s_{\text {sec }}^{\gamma}, \omega^{\mathrm{det}}=\bar{\omega} \pm C V_{\omega} \cdot s_{\omega},
$$

with $C V$ - critical value at level $\alpha . C V$ depends on the distribution of the statistic and on level of significance $\alpha$.

The values of boundaries and the size of the confidence intervals for kinematic quantities depends on:

- the direction in which they are treated,

- random errors in observations conditional to accuracy of used geodetic instruments and measuring methods and

- local geometry of the geodetic network were the point is situated (network geometry means geometric distribution of points and the observations in the network).

Taking into account that the confidence intervals are different in different directions then it is more appropriate to talk about confidence areas at chosen confidence level. The shape and the size of confidence areas for each kinematic quantity in certain point represents the detection level of deformations in the geodetic network, which is understood as the level of sensitivity to the strains and rotations in the geodetic network. The higher detection level in particular point, the smaller deformations geodetic network is able to detect. We want to establish (in a phase of establishing network) such a network geometry that the detection levels are as high as possible, and confidence areas in their sizes as small as possible with homogenous shape in all directions. 

distribution function. As it was shown by Savšek-Safić et al. (2006) a random variable, which is in nonlinear connections to normally distributed random variable is not normally distributed. Due to nonlinear functional relationship between normally distributed point displacements and kinematic quantities, the analytical derivation of probability distribution functions (Xu and Grafarend 1996) is very complex. The two main reasons to empirically derive probability distribution function are:

- the fact that in such case there is no need to involve any linearization to simplify the existing mathematical model, which connect displacements and kinematic quantities and

- possibility to use numerical methods to simulate large number of sample elements to obtain real properties of random variable.

Similar to confidence intervals the probability distributions is going to be studied separately for each direction (sector) in certain point. An example of empirically derived probability distributions and confidence areas of deformation parameters is shown in the sequel.

\section{Statistical significance of strains and rotations}

Known confidence areas for kinematic quantities in each point of geodetic network can serve us as useful tool to determine the statistical significance of deformation when it actually occurs. The statistical significant strains and rotation can be determined using statistical testing. We pose null hypothesis as a statement that there are no strains and rotation in geodetic network:

$$
\mathrm{H}_{0}: \varepsilon_{i}, \gamma_{i}, \omega_{i}=0 \text { (no strains and rotation in point } i \text { ). }
$$

We specify an alternative hypothesis:

$\mathrm{H}_{1}: \varepsilon_{i}, \gamma_{i}, \omega_{i} \neq 0$ (network has deformed or rotated in point $i$ ).

The statistical tests are carried out on the basis of known probability distributions of random variables, in this case the kinematic quantities. The computed value of deformation parameter represents the test statistics. If the value of test statistics is outside the boundaries of confidence area, then we reject the null hypothesis at the level of the significance $\alpha / 2$ of the selected test. We then say that deformation in point exists and is statistically significant at significance level $\alpha$. 


\section{Numerical example}

Detection level of deformations in the geodetic network is examined in the case of geodetic network in 2D (Figure 2). Points are located in the network in the way that we can assume that there is a part of the network with good geometry and a part of the network (near point 12), which is expected to be less sensitive to strains and rotations. Comparing the relative and absolute error ellipses (Figure 2), we can see that the geometry of the network is getting weak towards point 12 . Therefore, we expect the geodetic network to be less sensitive to strains and rotations in this area.

The Monte Carlo approach is used to simulate the number of sim sets of observed directions and distances in geodetic network. The sample size is of great importance to deduce real information about statistical property of random variable. The decision about sample size, used in simulations is made empirically. Since we are computing first and second order moments of kinematic quantities, we can easily compute these values after specific number of simulations. It is obvious from graph (Figure 3) that e.g. mean value converges to some value and therefore the sample size of $\operatorname{sim}=100000$ is large enough for apppropriate assessment of statistical properties.

For each simulated set of observations in the network we compute correction vector of approximate values of coordinate unknowns using the least squares adjustment of observations within the free network. Understanding that corrections as displacements, kinematic quantities in each point of the network can be computed. For all simulations we compute for each point a multitude of size sim of all associated kinematic quantities $(\varepsilon, \gamma, \omega)$ with their directions. To determine the shape and the size of confidence areas considered as detection level of deformations for each quantity it is important to define the critical interval with its critical values at predefined confidence level for each direction sector. Critical values at level $\alpha$ can be deduced from empirically obtained probability distribution function.

\section{Empirical determination of probability distribution of kinematic quantities}


Each direction sector contains the number of $\operatorname{sim}_{\text {sec }}$ computed values of particular kinematic quantity. With this multitude of values we can plot the shape of empirical probability distribution function. Since we doubt in the congruence of the probability distribution with the normal distribution, we proceed the Kolmogorov-Smirnov test for goodness of fit with the normal probability distribution. We pose null hypothesis (Lilliefors 1967):

$\mathrm{H}_{0}$ : random variable is normally distributed: $X \sim N\left(\mu_{x}, \sigma_{x}\right)$,

against the alternative hypothesis:

$\mathrm{H}_{1}$ : random variable is not normally distributed: $X \not N\left(\mu_{x}, \sigma_{x}\right)$.

The statistical testing for normality of probability distribution is performed for each point and each kinematic quantity with sample size $\operatorname{sim}_{\text {sec }}$ separately. Numerical results for point 12 in Table 1 show that in most direction the Kolmogorov-Smirnov test rejects the null hypothesis at the $1 \%$ significance level. Test cannot reject the null hypothesis for normality in directions with low frequency and low amplitude of computed principal normal strain. But as we can see, for these sectors the value of test statistic for Kolmogorov-Smirnov test with confidence $\alpha=1 \%$ is very tight to the appertaining critical value.

General ascertainments after performed Kolmogorov-Smirnov test for empirically derived probability distribution of kinematic quantities are (Table 1):

- Probability distribution of normal strain $\varepsilon$ and shear strain $\gamma$ depends on the direction of deformation in point.

Statistical tests rejects the normality in many direction at certain point. For shear strain in all direction the probability distribution is not normal.

- Rotation $\omega$, according to linear functional relationship to point displacements (equation (7)), is normally distributed (it applies to all points in the network).

Once we have empirical probability distribution function in some direction we can determine the boundaries of orientation-wised confidence interval. We use the interpolation method to determine the upper and the lower critical value at the selected level of confidence $1-\alpha$. The critical value $(C V)$ is then for standardized form computed as:

$$
C V_{j, i}^{\alpha / 2, \text { std }}=\frac{C V_{j, i}^{\alpha / 2}-\bar{j}_{i}}{s_{j, i}} \text { (for lower tail), } \quad C V_{j, i}^{1-\alpha / 2, \text { std }}=\frac{C V_{j, i}^{1-\alpha / 2}-\bar{j}_{i}}{s_{j, i}} \text { (for upper tail), }
$$


where $j=\varepsilon, \gamma, \omega$ and $i=1, \ldots, 12$.

The boundaries of confidence intervals in certain directions allow us to determine the edge of the confidence area for each point in the network and for both deformation parameter (normal and shear strain). From Figure 5 and Figure 6 it is obvious that confidence areas differ in shape and size from point to point. For points in hexagon 1-2-3-4-6-7 with central point 5 the confidence areas are quite small and in shape very homogenous in all directions (almost circles) if we consider either normal or shear strain. Towards point 12 confidence areas become very heterogenous, having two main directions with bigger boundary values. This means that the network near the point 12 is less sensitive to strains and rotations and has in that area weaker geometry.

Summarizing numerical results from Tables 1 and 2 and Figures 5 and 6, the detection level of deformation is relatively to other points much higher in areas with better network geometry, this is in area of hexagon 1-2-3-4-6-7. Consequently, any deformation, which occurs in geodetic network will be easily recognized as statistically significant in areas with higher detection level of deformations. Geodetic network with high detection level of deformations and homogenous shape confidence areas is therefore more appropriate for quality deformation analysis.

\section{CONCLUSION}

Deformations of an object, discretized by geodetic network, were considered as mechanical strains and rotation. We were computing the kinematic quantities from strain and rotation tensors, which are in a functional relationship to the vector field of point displacements. Because of the specific representation of deformable object by network points, the strains and rotation in the points were considered under the assumption that the strains and rotation between points are homogeneous. This is the only acceptable way, since we are dealing with discrete data of the vector field of displacements at the area (object or figure) i.e. with the points of geodetic network. We determined the strains and rotations from the known displacements of network points. The computations of kinematic quantities in particular point were not realized under the assumption of the differential closeness of neighbouring network points (from the theory of mechanics of solids). Thus, we introduced the so called weight matrix into computation of displacement gradient matrix. In this way we took into account the impact of strains and rotation of closer points. 
In order to reliably determine the statistical significance of strains and rotations, it is necessary to determine the probability distribution of kinematic quantities. Although many researchers dealt with this problem and analytically derived probability properties we used empirical approach. We empirically derived the probability distribution functions for normal and shear strain at different direction in each point of geodetic network using simulations of observations in the network. Based on the empirical determination of the probability distribution in certain direction, we found that:

- the probability distribution of each kinematic quantity (mean and standard deviation) depends on the direction in which it is treated and on the local geometry of the geodetic network,

- the principal strain is not normally distributed,

- rotation is normally distributed independently of the position of point in the network.

\begin{abstract}
The empirical probability distribution function allows us to determine orientation-wised confidence intervals, which together for all directions define the confidence area. The shapes and the sizes of confidence areas for the assumed measurements accuracy depend on the location in geodetic network and local geometry of geodetic network and are considered as detection level of deformations. The larger the confidence area of certain kinematic quantity, less is the network sensitive to deformations and is less able to identify lower values of strains and rotations. In the particular case of the geodetic network it is clear that the network in points with less connections between neighbouring points is less capable of detecting smaller values of strains and rotations.
\end{abstract}

The empirically defined statistical properties of kinematic quantities in individual point with known confidence areas can serve us as useful tool to determine statistical significant strains and rotations between two time epochs.

\title{
NOTATION
}

The following symbols are used in this paper:

$x, y, z=$ coordinate components;

$\mathbf{u}=$ vector of point displacements;

$u_{x}, u_{y}=$ components of vector $\mathbf{u}$;

$T_{i}, T_{j}=$ points; 
$i, j=$ indexes for points $T_{i}, T_{j}$;

$\varepsilon_{x x}, \varepsilon_{y y}, \varepsilon_{x y}=$ elements of tensors of small strains;

$\omega_{i}=$ differential rotation in point;

$\mathbf{r}_{i}, \mathbf{r}_{j}=$ coordinate vector of points $T_{i}$ and $T_{j} ;$

$\mathbf{r}_{i j}=$ coordinate differences of points $T_{i}$ and $T_{j}$;

$\mathbf{c}=$ constant vector;

$a_{i}, b_{i}=$ components of $\mathbf{c}$;

$\mathbf{E}_{i}=$ displacement gradient matrix;

$\mathbf{P}_{F}=$ weight matrix for computation of $\mathbf{E}_{i} ;$

$p_{i j}=$ weight for connection between $T_{i}$ and $T_{j} ;$

$d_{i j}=$ distance between points $T_{i}$ and $T_{j} ;$

$\varepsilon_{1,2}^{i}=$ principle normal strain;

$\gamma_{1,2}^{i}=$ principle shear strain;

$\operatorname{sim}=$ number of simulations;

$\bar{\varepsilon}, \bar{\gamma}, \bar{\omega}=$ mean values of kinematic quantities;

$s_{\varepsilon}, s_{\gamma}, s_{\omega}=$ standard deviations of kinematic quantitie;

$\varepsilon_{\mathrm{det}}, \gamma_{\mathrm{det}}, \omega_{\mathrm{det}}=$ detection level of deformations for each kinematic quantity;

$C V$ = critical value;

$d$ = point displacement;

$\Delta y, \Delta x=$ coordinate differencies;

$y_{0}, x_{0}=$ approximate values of point coordinates;

$y_{i}, x_{i}=$ computed coordinates of the points;

$\alpha=$ level of significance; 


\section{REFERENCES}

Berber, M. (2006). "Robustness Analysis of Geodetic Networks." Technical Report No 242, Department of Geodesy and Geomatics Engineering, University of New Brunswick, Fredericton, NB, Canada.

Berber, M., Dare, P., and Vaniček, P. (2006). "Robustness Analysis of Two-Dimensional Networks." J. Surv. Eng., 132(4), 168-175.

Cai, J., and Grafarend, E. (2007a)."The statistical analysis of geodetic deformation (strain rate) derived from the space geodetic measurements of BIFROST project in Fennoscandia" J. Geodyn., 43(2), 214-238.

Cai, J., and Grafarend, E. (2007b). "Statistical analysis of the eigenspace components of the two-dimensional, symmetric rank-two strain tensor derived from the space geodetic measurements (ITRF92 - ITRF2000 data set) in central Mediterranean and Western Europe." Geophys. J. Int., 168(1), 449-472.

Cai, J., Grafarend E., and Schaffrin B. (2005). "Statistical inference of the eigenspace components of a two-dimensional, symmetric rank two random tensor." J. Geodesy, 78 (7-8), 425-436.

Chrzanowski, A., Chen, Y. Q., and Secord, J. M. (1983). "Analysis of the Simulated Monitoring Network Using the Fredericton Approach." Deformationsanalysen '83, Geometrische Analyse und Interpretation von Deformationen Geodatischer Netze (ed. W. Welsch), Schriftenreihe, Wissenschaftlicher Studiengang Vermessungswesen, Hochschule der Bundeswehr Muenchen, Muenchen, Germany.

Lilliefors, H. W. (1967). "On the Kolmogorov-Smirnov test for normality with mean and variance unknown." J. Statistical Assn., 62(318), 399-402.

Michel, V., and Person, T. (2003). "From geodetic monitoring to deformation tensors and their reliability." Proc., $11^{\text {th }}$ FIG Symposium on Deformation Measurements, Santorini, Greece.

Mierlo, J. van (1978). "A testing procedure for analysing geodetic deformation measurements." Proc., 2nd FIG symposium on deformation measurements by geodetic methods, Bonn, Germany.

Mierlo, J. van (1980). "Free Network Adjustment and S - transformation." DGK, Karlsruhe, Reihe B, 252, 41-45.

Rubinstein, R. Y. (1981). "Simulation and the Monte Carlo method." Wiley, New York.

Savšek-Safić, S., Ambrožič, T., Stopar, B., Turk, G. (2006). "Determination of Point Displacements in the Geodetic Network." J. Surv. Eng., 132(2), 58-63. 
Shames, I. H., and Cozzarelli, F. A. (1997). "Elastic and Inelastic Stress Analysis." Taylor\&Francis, Revised

Printing, Washington.

Soler, T., and Van Gelder, B. H. W. (1991). "On covariances of eigenvalues and eigenvectors of second-rank symmetric tensors." Geophys. J. Int., 105(2), 537-546

Soler, T., and Van Gelder, B. H. W. (2006). "Corrigendum: On covariances of eigenvalues and eigenvectors of second-rank symmetric tensors." Geophys. J. Int., 165(1), 382-382.

Stopar, B. (2001). "Second order design of horizontal GPS net." Surv. Rev., 36 (279), 44-53.

Vaníček, P., Craymer, M. R., and Krakiwsky, E. J. (2001). "Robustnes analysis of geodetic horizontal networks." J. Geodesy, 75(4), 199-209.

Vaniček, P., Grafarend, E. W., and Berber, M. (2008). "Strain invariants." J. Geodesy, 82(4-5), 263-268.

Vaniček, P., and Krakiwsky, E. J. (1986). "Geodesy: The Concepts." 2nd edn., North Holland/Elsevier, Amsterdam. Welsch, W., and Zhang, Y. (1983). "Einige Methoden zur Untersuchung kongruenter und Affiner Beziehungen in Geodatischen Unberwacungsnetzen zur Ermittlung von Deformationen." Deformationsanalysen '83, Geometrische Analyse und Interpretation von Deformationen Geodatischer Netze (ed. W. Welsch), Schriftenreihe, Wissenschaftlicher Studiengang Vermessungswesen, Hochschule der Bundeswehr Muenchen, Muenchen, Germany. Xu, P., and Grafarend, E. (1996). "Probability distribution of eigenspectra and eigendirections of a twodimensional, symmetric rank two random tensor." J. Geodesy, 70(7), 419-430.

Xu, P., Schimada, S., Fujii, Y., and Tanaka, T. (2000). "Invariant geodynamical information in geometric geodetic measurements." Geophys. J. Int., 142(2), 586-602. 
Table 1 and 2

\section{Click here to download Table: tables_rev.doc}

1

3

4

5

6

7

8

9

10

11

12

13

14

15

16

17

18

19

20

21

22

23

24

25

26

27

28

29

30

31

32

33

34

35

36

37

38

39

40

41

42

43

44

45

46

47

48

49

50

51

52

53

54

55

56

57

58

59

60

61

62

63

64

65

Table 1

Statistical properties of principal normal strain in point 12 .

\begin{tabular}{|c|c|c|c|c|c|c|c|c|c|c|}
\hline \multirow{2}{*}{$\begin{array}{c}\begin{array}{c}\text { Point } \\
12\end{array} \\
\text { Sector } \\
\end{array}$} & \multicolumn{3}{|c|}{$\begin{array}{c}\text { Kolmogorov-Smirnov test } \\
\qquad \alpha=1 \% \\
\end{array}$} & \multicolumn{2}{|c|}{$\begin{array}{c}\text { confid. interval - CI } \\
\alpha=5 \% \\
\end{array}$} & \multicolumn{2}{|c|}{$\begin{array}{c}\text { stand. critical value }-C V \\
\quad \alpha=5 \%\end{array}$} & \multicolumn{2}{|c|}{$\begin{array}{l}1^{\text {st }} \& 2^{\text {nd }} \text { order } \\
\text { moments }\end{array}$} & \multirow[b]{2}{*}{$\begin{array}{c}\text { mean direction } \\
\text { for sector } \\
{\left[{ }^{\circ}\right]}\end{array}$} \\
\hline & reject $\mathrm{H}_{0}$ ? & $\begin{array}{l}\text { test } \\
\text { statistic }\end{array}$ & $\begin{array}{l}\text { critical } \\
\text { value }\end{array}$ & $\begin{array}{c}\text { lower } \\
\text { boundary } \\
\text { [ppm] }\end{array}$ & $\begin{array}{c}\text { upper } \\
\text { boundary } \\
{[\mathrm{ppm}]}\end{array}$ & lower tail & upper tail & $\begin{array}{c}\text { mean } \\
{[\mathrm{ppm}]}\end{array}$ & $\begin{array}{c}\text { standard } \\
\text { deviation } \\
{[\mathrm{ppm}]}\end{array}$ & \\
\hline 1 & yes & 0.0302 & 0.0169 & -20.3219 & 20.5433 & -1.8491 & 1.8832 & -0.0759 & 10.9493 & 4.5 \\
\hline 2 & yes & 0.0246 & 0.0190 & -18.5970 & 17.9309 & -1.9182 & 1.8501 & -0.0030 & 9.6934 & 13.5 \\
\hline 3 & yes & 0.0298 & 0.0195 & -17.6320 & 17.0450 & -1.8876 & 1.8390 & -0.0674 & 9.3055 & 22.5 \\
\hline 4 & yes & 0.0257 & 0.0186 & -16.9184 & 16.8615 & -1.8641 & 1.9110 & -0.2385 & 8.9480 & 31.5 \\
\hline 5 & yes & 0.0182 & 0.0159 & -17.3420 & 16.2671 & -1.9973 & 1.8848 & -0.0503 & 8.6575 & 40.5 \\
\hline 6 & yes & 0.0202 & 0.0119 & -17.1915 & 17.1410 & -1.8739 & 1.8694 & -0.0044 & 9.1718 & 49.5 \\
\hline 7 & yes & 0.0217 & 0.0076 & -19.4050 & 19.1882 & -1.8772 & 1.8636 & -0.0384 & 10.3168 & 58.5 \\
\hline 8 & yes & 0.0206 & 0.0059 & -18.9558 & 19.2445 & -1.8702 & 1.8889 & 0.0493 & 10.1621 & 67.5 \\
\hline 9 & no & 0.0052 & 0.0084 & -14.1234 & 14.1438 & -1.9315 & 1.9429 & -0.0317 & 7.2959 & 76.5 \\
\hline 10 & no & 0.0060 & 0.0127 & -11.9871 & 12.1811 & -1.9465 & 1.9401 & 0.1166 & 6.2184 & 85.5 \\
\hline 11 & no & 0.0107 & 0.0169 & -11.7915 & 11.2080 & -1.9826 & 1.8857 & -0.0037 & 5.9457 & 94.5 \\
\hline 12 & no & 0.0152 & 0.0190 & -12.0383 & 11.2802 & -2.0274 & 1.9113 & -0.0352 & 5.9204 & 103.5 \\
\hline 13 & no & 0.0169 & 0.0195 & -12.2625 & 12.0456 & -2.0034 & 1.9864 & -0.0568 & 6.0926 & 112.5 \\
\hline 14 & no & 0.0081 & 0.0186 & -12.3151 & 12.6863 & -1.9218 & 1.9744 & 0.0167 & 6.4169 & 121.5 \\
\hline 15 & yes & 0.0187 & 0.0159 & -14.3416 & 14.6173 & -1.9031 & 1.9627 & -0.0854 & 7.4912 & 130.5 \\
\hline 16 & yes & 0.0202 & 0.0119 & -18.8744 & 18.6719 & -1.9054 & 1.8739 & 0.0553 & 9.9349 & 139.5 \\
\hline 17 & yes & 0.0437 & 0.0076 & -30.8835 & 30.5242 & -1.8408 & 1.8132 & 0.0524 & 16.8057 & 148.5 \\
\hline 18 & yes & 0.0647 & 0.0059 & -39.9685 & 40.2605 & -1.7437 & 1.7707 & -0.1622 & 22.8286 & 157.5 \\
\hline 19 & yes & 0.0509 & 0.0084 & -33.6691 & 33.4745 & -1.7989 & 1.8055 & -0.1583 & 18.6284 & 166.5 \\
\hline 20 & yes & 0.0461 & 0.0127 & -24.3771 & 24.4871 & -1.8314 & 1.8176 & 0.1472 & 13.3914 & 175.5 \\
\hline
\end{tabular}

Table 2

Statistical properties of principal shear strain in point 12 .

\begin{tabular}{|c|c|c|c|c|c|c|c|c|c|c|}
\hline \multirow{2}{*}{$\begin{array}{c}\begin{array}{c}\text { Point } \\
12\end{array} \\
\text { Sector }\end{array}$} & \multicolumn{3}{|c|}{$\begin{array}{c}\text { Kolmogorov-Smirnov test } \\
\alpha=1 \%\end{array}$} & \multicolumn{2}{|c|}{$\begin{array}{c}\text { Confid. interval }-C I \\
\alpha=5 \%\end{array}$} & \multicolumn{2}{|c|}{$\begin{array}{c}\text { Stand. critical value }-C V \\
\quad \alpha=5 \%\end{array}$} & \multicolumn{2}{|c|}{$\begin{array}{c}1^{\text {st }} \& 2^{\text {nd }} \text { order } \\
\text { moments }\end{array}$} & \multirow[b]{2}{*}{$\begin{array}{c}\text { mean direction } \\
\text { for sector } \\
{\left[{ }^{\circ}\right]}\end{array}$} \\
\hline & reject $\mathrm{H}_{0}$ ? & $\begin{array}{c}\text { test } \\
\text { statistic }\end{array}$ & $\begin{array}{l}\text { critical } \\
\text { value }\end{array}$ & $\begin{array}{c}\text { lower } \\
\text { boundary } \\
\text { [ppm] }\end{array}$ & $\begin{array}{c}\text { upper } \\
\text { boundary } \\
{[\mathrm{ppm}]}\end{array}$ & lower tail & upper tail & $\begin{array}{l}\text { mean } \\
{[\mathrm{ppm}]}\end{array}$ & $\begin{array}{c}\text { standard } \\
\text { deviation } \\
{[\mathrm{ppm}]}\end{array}$ & \\
\hline 1 & yes & 0.0815 & 0.0119 & -13.4547 & 13.4670 & -1.7466 & 1.7559 & -0.0298 & 7.6864 & 4.5 \\
\hline 2 & yes & 0.0764 & 0.0076 & -21.2929 & 21.5586 & -1.7477 & 1.7770 & -0.0454 & 12.1575 & 13.5 \\
\hline
\end{tabular}




\begin{tabular}{|c|c|c|c|c|c|c|c|c|c|c|}
\hline 3 & yes & 0.0847 & 0.0059 & -26.3888 & 26.3880 & -1.7391 & 1.7252 & 0.1057 & 15.2344 & 22.5 \\
\hline 4 & yes & 0.0778 & 0.0084 & -19.6717 & 19.5354 & -1.7774 & 1.7537 & 0.0633 & 11.1036 & 31.5 \\
\hline 5 & yes & 0.0859 & 0.0127 & -12.4080 & 12.3344 & -1.7342 & 1.7282 & -0.0153 & 7.1459 & 40.5 \\
\hline 6 & yes & 0.0852 & 0.0169 & -9.3203 & 9.1689 & -1.7510 & 1.7092 & 0.0361 & 5.3434 & 49.5 \\
\hline 7 & yes & 0.0882 & 0.0190 & -7.9683 & 8.2121 & -1.6986 & 1.7576 & -0.0161 & 4.6816 & 58.5 \\
\hline 8 & yes & 0.0895 & 0.0195 & -7.8890 & 7.8775 & -1.7209 & 1.7160 & 0.0053 & 4.5874 & 67.5 \\
\hline 9 & yes & 0.0862 & 0.0186 & -8.3592 & 8.3091 & -1.7623 & 1.6989 & 0.1276 & 4.8157 & 76.5 \\
\hline 10 & yes & 0.0885 & 0.0159 & -9.6660 & 9.8980 & -1.7120 & 1.7594 & -0.0175 & 5.6358 & 85.5 \\
\hline 11 & yes & 0.0815 & 0.0119 & -13.4720 & 13.4499 & -1.7566 & 1.7459 & 0.0298 & 7.6864 & 94.5 \\
\hline 12 & yes & 0.0764 & 0.0076 & -21.5858 & 21.2865 & -1.7793 & 1.7472 & 0.0454 & 12.1575 & 103.5 \\
\hline 13 & yes & 0.0847 & 0.0059 & -26.4039 & 26.3854 & -1.7262 & 1.7389 & -0.1057 & 15.2344 & 112.5 \\
\hline 14 & yes & 0.0778 & 0.0084 & -19.5455 & 19.6659 & -1.7546 & 1.7768 & -0.0633 & 11.1036 & 121.5 \\
\hline 15 & yes & 0.0859 & 0.0127 & -12.3397 & 12.3985 & -1.7290 & 1.7329 & 0.0153 & 7.1459 & 130.5 \\
\hline 16 & yes & 0.0852 & 0.0169 & -9.1741 & 9.3133 & -1.7102 & 1.7497 & -0.0361 & 5.3434 & 139.5 \\
\hline 17 & yes & 0.0882 & 0.0190 & -8.2301 & 7.9636 & -1.7614 & 1.6976 & 0.0161 & 4.6816 & 148.5 \\
\hline 18 & yes & 0.0895 & 0.0195 & -7.8802 & 7.8836 & -1.7166 & 1.7197 & -0.0053 & 4.5874 & 157.5 \\
\hline 19 & yes & 0.0862 & 0.0186 & -8.3346 & 8.3469 & -1.7042 & 1.7598 & -0.1276 & 4.8157 & 166.5 \\
\hline 20 & yes & 0.0885 & 0.0159 & -9.9045 & 9.6474 & -1.7605 & 1.7087 & 0.0175 & 5.6358 & 175.5 \\
\hline
\end{tabular}




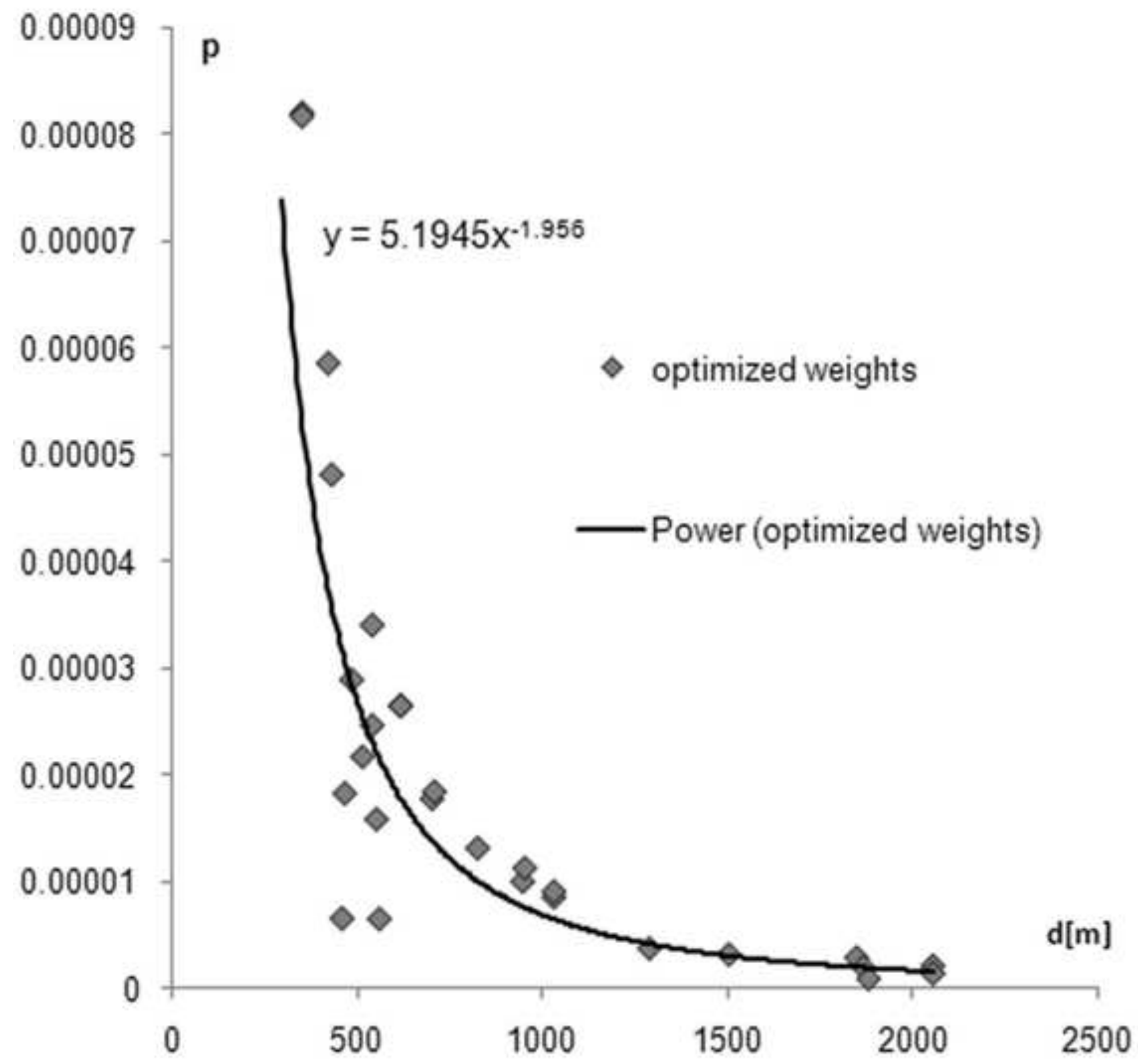


Figure 2

Click here to download high resolution image

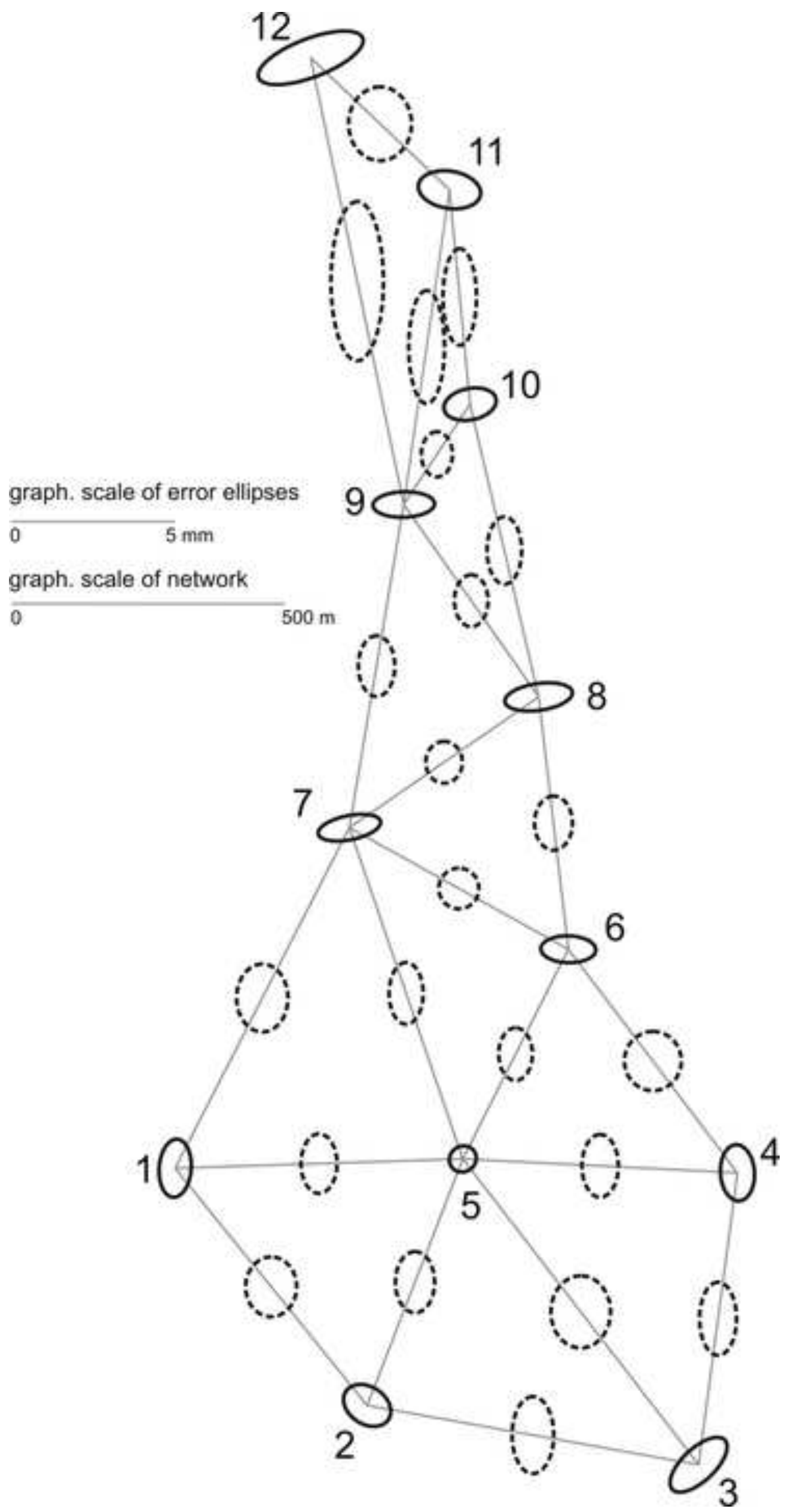




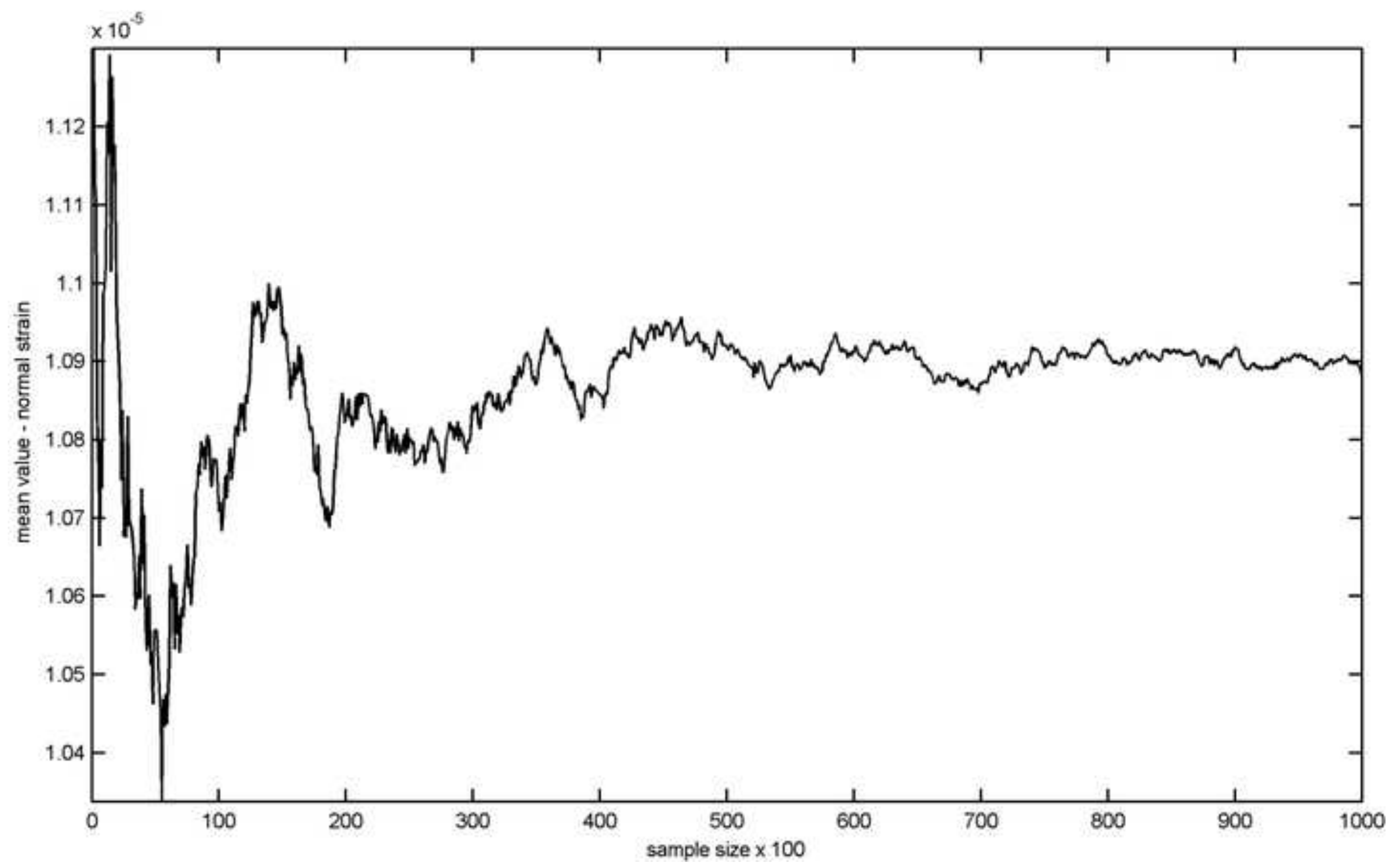




\section{Figure 4}

Click here to download high resolution image

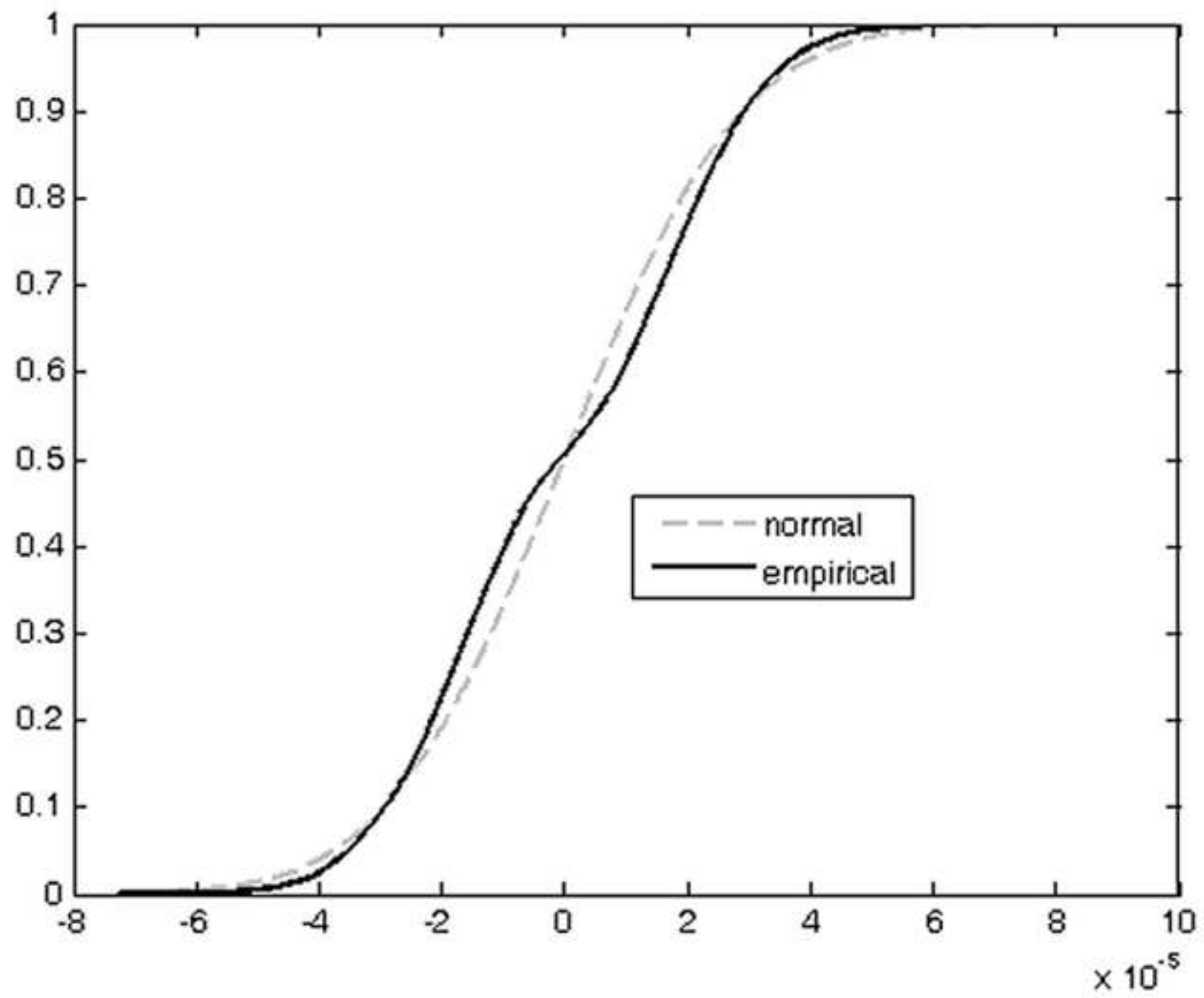


Figure 5

Click here to download high resolution image

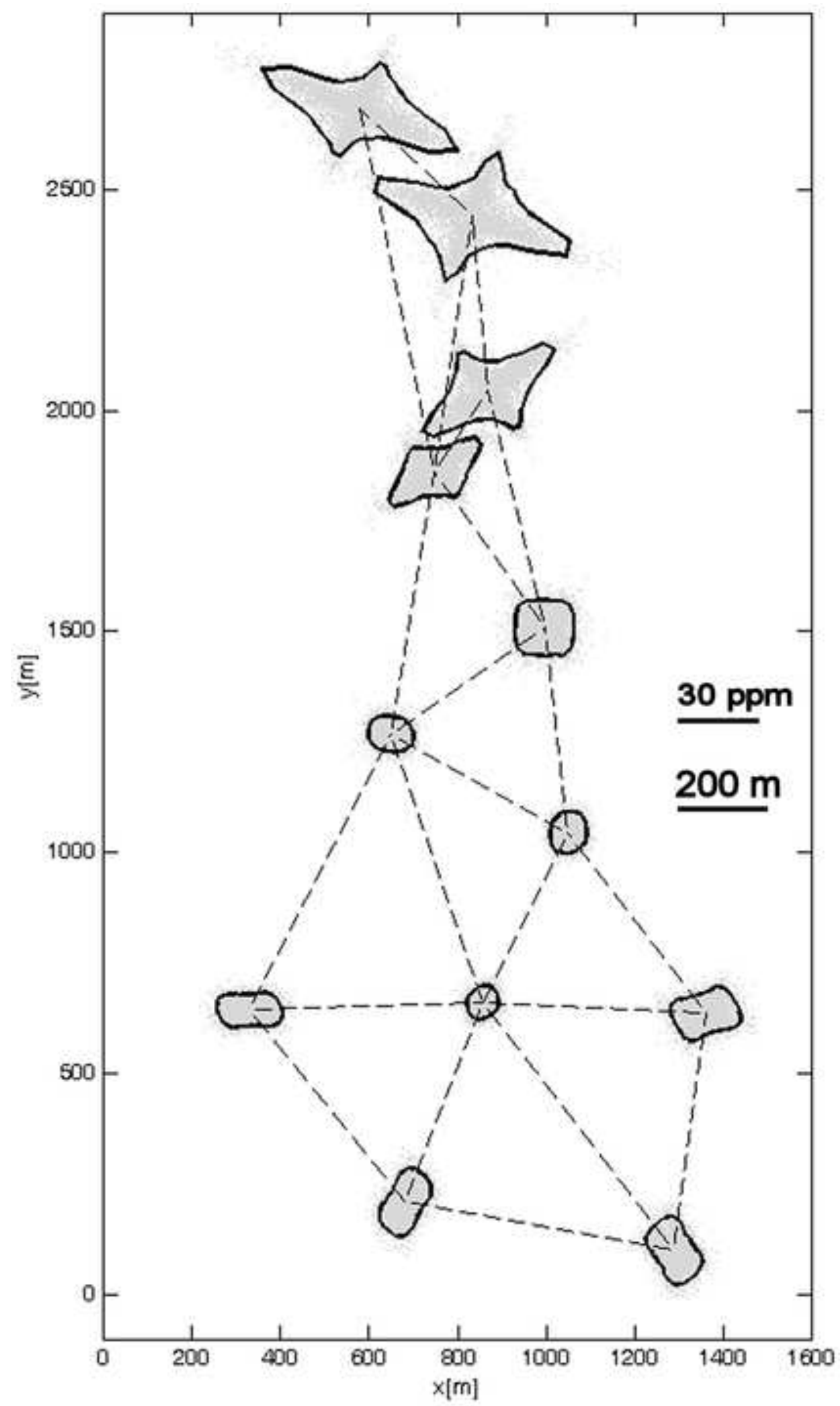


Figure 6

Click here to download high resolution image

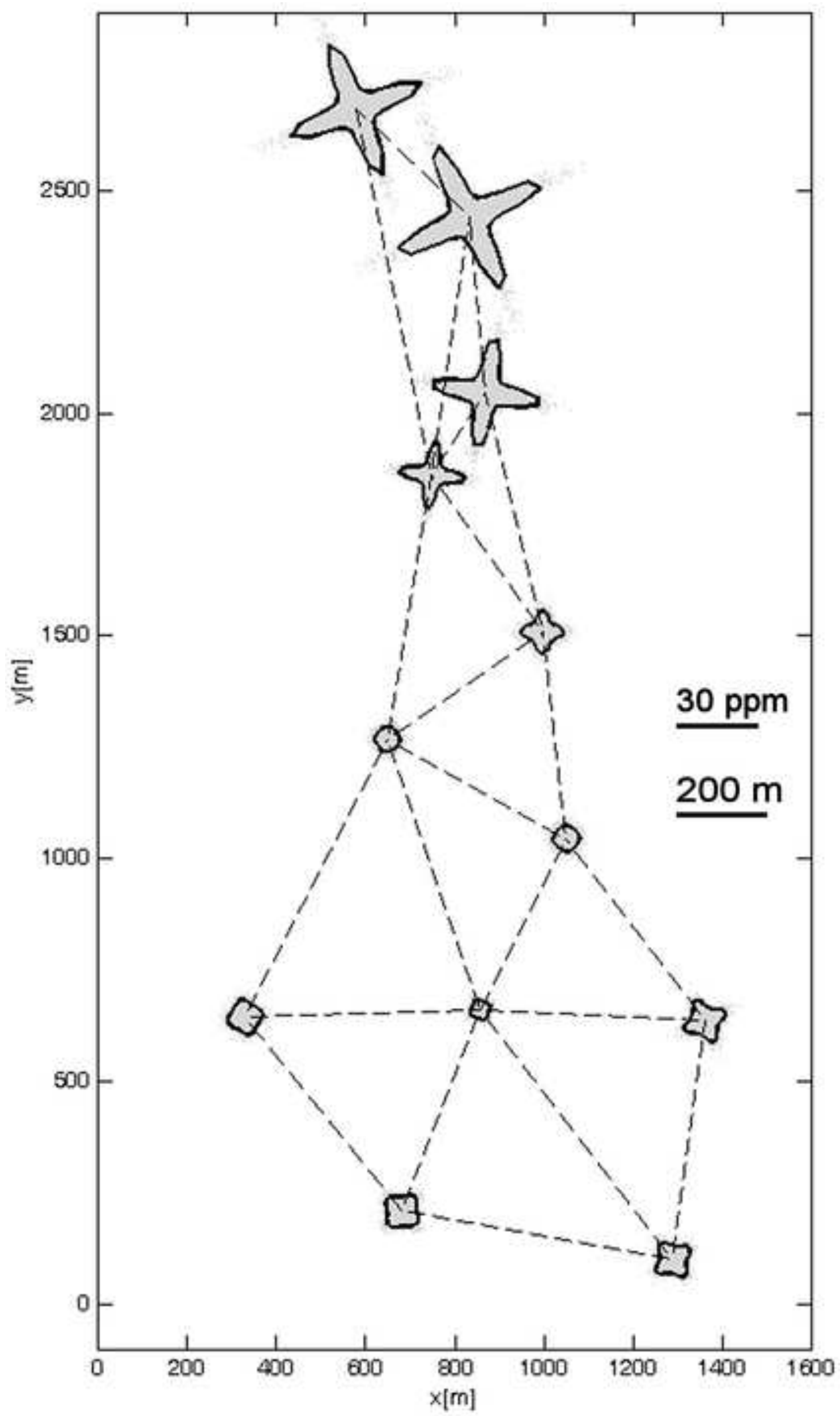




\section{FIGURES}

Figure 1

Functional relation of weight $p_{i j}$ and distance between points.

Figure 2

Geodetic network of 12 points with measurement connections and absolute and relative error ellipses.

Figure 3

Convergence of mean value of principal normal strain in function of sample size.

Figure 4

Probability distribution function for principal normal strain (point 12, direction $157^{\circ}$ )

Figure 5

95\% confidence areas for principal normal strain.

Figure 6

95\% confidence areas for principal shear strain. 